\title{
Time-Fractional Landau-Khalatnikov Model Applied To Numerical Simulation of Polarization Switching In Ferroelectrics
}

\author{
Anna Maslovskaya ( $\nabla$ maslovskayaag@mail.ru ) \\ Amur State University https://orcid.org/0000-0002-5628-3519 \\ Lubov Moroz \\ Amur State University
}

\section{Research Article}

Keywords: Landau-Khalatnikov model, Ferroelectrics switching, Time fractional partial differential equation, Caputo derivative, Iterative finite difference scheme

Posted Date: January 24th, 2022

DOI: https://doi.org/10.21203/rs.3.rs-1252296/v1

License: (c) (i) This work is licensed under a Creative Commons Attribution 4.0 International License.

Read Full License 


\title{
Time-fractional Landau - Khalatnikov model applied to numerical simulation of polarization switching in ferroelectrics
}

\author{
Anna Maslovskaya - Lubov Moroz
}

Received: date / Accepted: date

\begin{abstract}
Ferroelectrics are complex structured media that exhibit fractal properties and memory effects in terms of a number of dynamic characteristics. The most relevant applications of ferroelectrics in science and technology are associated with the primary mechanisms of polarization switching and domain structure dynamics induced by external exposure. In this study, we propose a time-fractional modification of the Landau - Ginzburg - Devonshire - Khalatnikov model to describe the dynamics of ferroelectric polarization switching. To solve the time-fractional cubic-quintic partial differential equation numerically, a computational scheme is derived. The technique combines an iterative procedure and an implicit finite-difference scheme based on an approximation of Caputo derivative. A series of computational experiments are presented to visualize polarization switching characteristics on the example of thin films of barium titanate. A variation of the order of fractional derivative as a numerical characteristic of the memory effect allows one to "adjust" suitable regimes of simulated dynamical system. The obtained findings indicate that the generalized time-fractional model can provide better reproduction of experimental data in comparison with the classical analogue.
\end{abstract}

Keywords Landau - Khalatnikov model · Ferroelectrics switching - Timefractional partial differential equation $\cdot$ Caputo derivative $\cdot$ Iterative finite difference scheme

This work was supported by the Russian Foundation for Basic Research (project no. 20-3190075).

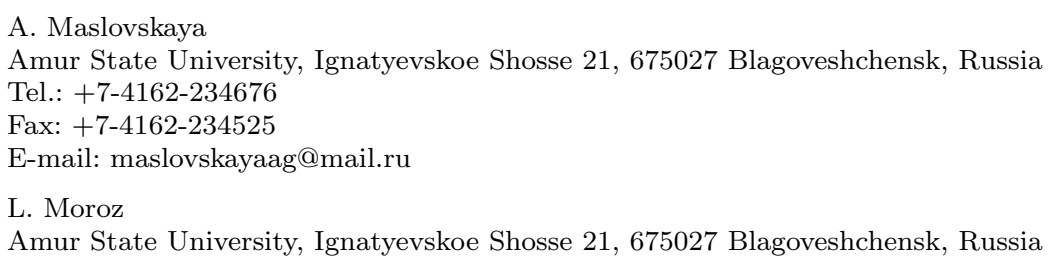


Mathematics Subject Classification (2020) 37M05 - 35R11 · 65M06

\section{Introduction}

Nowadays fractional differential equations are widely used for mathematical modeling of dynamic responses of complex structured physical media arising under non-equilibrium conditions [1-5]. to as non-classical, or anomalous. Generally, memory effects in physical systems can be modelled using timefractional partial differential equations (PDEs), whereas space-fractional PDEs are applied to describe dynamical processes in materials with nonhomogeneous structure and multiphase composition. Solid structures, disordered, random, and amorphous media, colloid materials are examples of such systems, which exhibit a complex geometry of bulk structure and surface topography, as well as a fractal behaviour of characteristics of dynamical responses $[6,7]$.

Specifically, ferroelectrics as a special class of promising polar dielectrics demonstrate complex scaling of domain configurations, self-similarity of nucleation processes and memory effects during the process of polarization switching, and common fractal properties of dynamical responses of crystals. Fractal features of domain structures, dielectric responses, and characteristics of polarization switching in ferroelectrics have been found by a number of independent researchers. Fractal analysis can be considered as a methodology for the study of the nature of ferroelectric phenomena and their most important properties, such as dielectric permittivity, light scattering, dielectric losses, hysteresis, acoustic emission, etc. Numerous works have established that domain configurations of ferroelectric systems possess both fractal structures and a self-similar character of nucleation and development of domains. These studies are based on methods of fractal and multifractal analysis of raster microscopic images of domain structures and time series presented by polarization responses [8-19].

Despite the fact that in recent decades the properties of self-similarity of the geometry of domain configurations and the memory effects in ferroelectrics have been intensively studied using the methods of fractal analysis, the application of the apparatus of fractional differential calculus is not so developed. At the same time it is obvious that the differential approach allows one to explore and predict the dynamical characteristics of the physical system. Certain fractional differential models have been proposed by independent research groups earlier to examine dynamic responses in ferroelectrics. For instance, the modification of the Kolmogorov-Avrami model for the estimation of polarization switching current in ferroelectrics has been developed on the basis of analytical calculation of fractional derivative in [20]. The fractional relaxation and oscillation equations have been introduced for ferroelectrics in [21,22]. Moreover, the fractional analogue of the Cole-Cole equation describing the dielectric relaxation has been considered in [23]. This approach of solving the problem has involved the use of finite-difference approximations of fractional derivatives. The similar numerical technique has been applied to implement the quasi-static model of ferroelectric hysteresis in [24]. The fractional order 
derivatives have been introduced to describe the non-linear mechanical and electric behavior of ferroelectric polymer composites in [25]. Moreover, a numerical solution of an fractional ordinary differential equation underlies the model of polarization switching current induced in ferroelectrics by electron beam irradiation $[26,27]$.

The most significant applications of ferroelectrics in science and technology are associated with the general mechanisms of polarization switching and dynamics of domain structures. A challenging problem which arises in this field is predicting polarization responses induced by external exposure in ferroelectric materials. The theoretical description of characteristics of ferroelectric polarization switching is supported by the framework of the Landau - Ginzburg Devonshire phenomenological theory. The thermodynamic formalism for ferroelectrics have been reported in many reviews $[28,29]$. The Landau theory of phase transitions has been previously used for modeling of polarization reversal processes and ferroelectric hysteresis loops in [30]. The corollaries of the Landau - Ginzburg - Devonshire theory can be employed to estimate the ferroelectric characteristics by means of the Ising and Heisenberg models implemented with the use of Monte-Carlo simulation and Metropolis algorithm $[31,32]$.

Furthermore, the time-dependent Landau - Khalatnikov equation has been proposed to describe the ferroelectric state and dynamics of polarization reversal under external field [28]. This model has attract more attention in the field of study of switching characteristics and hysteresis phenomena in ferroelectric thin films [31,33], the partial switching and external stress effect $[34,35]$, the peculiarities of ferroelectric phase transitions in the view of electrocaloric and pyroelectric effects [36], etc.

Generally, the mentioned approach is based on the mathematical apparatus of differential equations. In the present study we will focus on the Landau Ginzburg - Devonshire - Khalatnikov model, described by the initial-boundary value problem for cubic-quintic partial differential equation (PDE). Our previous study has been devoted to the exploring this model from both theoretical and computational points of view [37]. As shown above, the use of the fractional differential concept provides a generalization of integer models and significantly expands the range of functional capabilities of the mathematical modeling of physical systems. Since ferroelectrics possess complex polarization structures and demonstrate memory effects, we propose a time-fractional modification of the Landau - Ginzburg - Devonshire - Khalatnikov model to describe the polarization switching processes in ferroelectrics. This approach gives a significant advantage because it allows us to use an additional "flexible tool" for modeling of the space-time distribution of polarization in ferroelectrics and predicting dynamic responses. Obviously, analytical solutions of semilinear fractional PDEs meet serious problems. In the context of specific applied problems numerical methods are usually used, in particular, computational schemes based on the finite-difference approximations of fractional derivatives $[38,39]$. The preliminary result has been reported in [40], where we applied the simplified computational scheme and solved numerically the 
test-problem related to the generalized Landau - Khalatnikov model (without the using units of physical quantities).

The current study was undertaken to develop the Landau - Ginzburg Devonshire - Khalatnikov model and the computational algorithm for its implementation with application to the study of polarization switching characteristics and hysteresis in ferroelectric materials. The theoretical basis and governing equations of the generalized Landau - Khalatnikov model describing the polarization states of ferroelectrics will be presented in Section 2. Section 3 will be devoted to the construction of computational scheme for solving cubic-quintic fractional PDE. The Section 4 will focus on the computer simulation of polarization switching characteristics for typical ferroelectrics with the first-order phase transition. Here the performing full cycle of mathematical modeling and computer simulation to solve multidisciplinary problem also underlies the main significance of the present study. Finally, in Section 5, we will summarize main findings and present concluding remarks arising from this study.

\section{General description of the mathematical model}

In this section the mathematical problem statement designed for modeling of polarization switching in ferroelectrics is presented. The emphasis is placed on case of time-fractional modification of thermodynamic model describing dynamical responses in ferroelectrics .

A theoretical analysis of the properties of polarization switching and phase transitions in ferroelectrics can be performed on the basis of the Landau Ginzburg - Devonshire thermodynamic theory [29]. The role of the thermodynamic approach in the study of ferroelectrics can hardly be overestimated. The the Landau - Ginzburg - Devonshire theory enables describing the dielectric, pyroelectric, piezoelectric, electrocaloric and other properties of ferroelectric materials, and in many cases it gives a satisfactory agreement with the experimental results.

2.1 Thermodynamic framework for modeling of polarization switching in ferroelectrics

According to the concepts of thermodynamic theory, the equilibrium state of ferroelectrics can be completely specified by a fixed set of quantities: polarization, electric field, temperature, mechanical deformation and stress. The state of the ferroelectric material is determined by the free energy function and the values of the dependent variables correspond to the minimum free energy for the equilibrium state of the system. In a particular case, when there are no applied mechanical stresses, the polarization $P$ is directed along one of the crystallographic axes only. For uniaxial ferroelectrics, the free energy density $F$ can be expanded in powers of a single polarization component given by the following ansatz [29]: 


$$
F=\frac{1}{2} A P^{2}+\frac{1}{4} B P^{4}+\frac{1}{6} C P^{6}-E P,
$$

where $P$ is the spontaneous polarization in $\mathrm{C} / \mathrm{m}^{2} ; E$ is the field intensity in $\mathrm{V} / \mathrm{m} ; A=A_{0}\left(T-T_{C W}\right), A_{0}, B, C$ are the thermodynamic constants $(A$ in $\mathrm{m} / \mathrm{F} ; B$ in $\mathrm{m}^{5} /\left(\mathrm{C}^{2} \cdot \mathrm{F}\right) ; C$ in $\mathrm{m}^{9} /\left(\mathrm{C}^{4} \cdot \mathrm{F}\right) ; T$ is the current temperature and $T_{C W}$ is the Curie - Weiss temperature in $\left.\mathrm{K}\right)$.

The total free energy is calculated as $G=\int_{V} F d V$ in $\mathrm{J}$. The change in the free energy between the paraelectric phase at $T>T_{C W}$ and the ferroelectric phase at $T<T_{C W}$ depends on the coefficient $B$. If $B>0$, the second-order phase transition occurs, whereas $B<0$ is related to the first-order phase transition. For the second-order phase transition, the term containing the sixth power in the expression for the free energy density (1) can be neglected. The values of the thermodynamic parameters of $A_{0}$ and $C$ are positive for all known ferroelectrics [29]. Hereinafter for definiteness, we will consider ferroelectrics with the first-order phase transitions.

Further, we assume that polarization switching is induced by applying a periodical electric field

$$
E(t)=E_{0} \sin (\omega t)
$$

where $\omega=2 \pi f$ is the radial frequency of the applied field in $\mathrm{rad} / \mathrm{s}$ and $f$ is the frequency of field oscillations in $\mathrm{Hz}$.

Isaak Khalatnikov has developed the thermodynamic approach to describe the dynamics of ferroelectric polarization under an external field [28]:

$$
\delta \frac{d P}{d t}=-\frac{\partial F}{\partial P}
$$

where $\delta$ is the kinetic coefficient in $\mathrm{m} \cdot \mathrm{s} / \mathrm{F}$.

The proposed equation is known as the nonstationary Landau - Khalatnikov equation. The general Landau approach is based on the self-consistent field theory and, as a consequence, the relation (1) does not considers the fluctuations of polarization. As proposed by Ginzburg, including the gradient term or correlation energy $\psi \Delta P$ into the expression for free energy leads to the generalized analogue of the Landau - Khalatnikov equation given as follows:

$$
\frac{\partial P}{\partial t}=D \Delta P+\bar{A} P+\bar{B} P^{3}-\bar{C} P^{5}+\bar{\sigma} E(t),
$$

where $D=\psi / \delta$ is the thermodynamic parameter as combination of the gradient coefficient $\psi$ and the kinetic coefficient $\delta$, with the sense and the dimension of the diffusion coefficient in $\mathrm{m}^{2} / \mathrm{s} ; \bar{\sigma}=\nu / \delta ; \nu$ is the scaling factor; $\bar{A}=-A / \delta$, $\bar{B}=-B / \delta, \bar{C}=C / \delta$ are the positive constants.

The ferroelectric hysteresis loop can be estimated for certain values of the temperature $T$ as a polarization - electric field dependence calculated at successive times. In this way, the generalized Landau-Khalatnikov equation (4) and the periodical field intensity defined by (2) underlie the mathematical model of ferroelectric polarization switching in uniaxial ferroelectrics. A suitable set of boundary conditions and initial condition should be posed to close 
the mathematical problem. As will be shown, the specific form of the boundary conditions is due to the physical meaning of corresponding particular problems.

\subsection{Mathematical problem statement}

In view of the complex nature of the domain structure rearrangement and memory effects observed during polarization switching in ferroelectrics, we introduce into consideration the time-fractional modifications of the Landau - Ginzburg - Devonshire - Khalatnikov equation. The new generalization of the thermodynamic approach is bearing in mind the variation of dynamical regimes as well as their model representations that are associated with time dispersion processes arising in ferroelectric materials.

Let us consider the electric field applied parallel to the general direction of polarization $P$. In this case, the polarization $P$ is an order parameter, which depends on one space variable $x$. The surfaces of ferroelectric sample are specified by $x=0$ and $x=L$. Note that the polarization and the applied electric field are vector quantities. This means that only two possible states of polarization $\uparrow \mathbf{P}$ and $\downarrow \mathbf{P}$ are modeled during a complete switching of polarization by $180^{\circ}$, associated with the orientation of the applied field $\uparrow \mathbf{E}$ and $\downarrow \mathbf{E}$, respectively.

The time-fractional analogue of the Landau - Ginzburg - Devonshire Khalatnikov model is represented as an initial-boundary value problem for the following semilinear fractional partial differential equation:

$$
\begin{gathered}
\frac{\partial^{\alpha} P}{\partial \tau^{\alpha}}=d \frac{\partial^{2} P}{\partial x^{2}}+a P+b P^{3}-c P^{5}+\sigma E, \quad 0<x<L, \quad 0<\tau \leq \theta \backslash t^{*}, \\
\left.P\right|_{t=0}=P_{0}(x), \quad 0<x<L, \\
-\left.\frac{d P}{d x}\right|_{x=0}+\left.q P\right|_{x=0}-g=0,\left.\quad \frac{d P}{d x}\right|_{x=L}+\left.q P\right|_{x=L}-g=0, \quad 0<\tau \leq \theta \backslash t^{*},
\end{gathered}
$$

where $d=D t^{*} ; a=\bar{A} t^{*} ; b=\bar{B} t^{*} ; c=\bar{C} t^{*} ; \sigma=\bar{\sigma} t^{*}$ are the combinations of model parameters; $\tau=t \backslash t^{*}$ is the dimensionless time; $t^{*}$ is the characteristic time of the process in s; $q$ and $g$ are model parameters; $\theta$ is the time of process observation in s; $L$ is the thickness of the film in $\mathrm{m}$; $\alpha$ is the order of timefractional derivative, $0<\alpha<1$.

The characteristic time $t^{*}$ is introduced to provide an agreement of the physical dimensions and units of measure of variables and parameters of the model. In order to analyze the results of computer simulations, the reverse conversion is required (from the dimensionless time $\tau$ to the dimensional analogue $t)$. 
Within the framework of the current study, the mathematical problem statement (5)-(7) allows us to consider two principle problems. First, the model of polarization switching in ferroelectric thin films can be specified at zero initial condition $P_{0}(x)=0$ and the boundary conditions (7) should be transformed to the following view [29]:

$$
\left.\frac{d P}{d x}\right|_{x=0}=\left.\frac{P}{\lambda}\right|_{x=0},\left.\quad \frac{d P}{d x}\right|_{x=L}=-\left.\frac{P}{\lambda}\right|_{x=L}, \quad 0<\tau \leq \theta \backslash t^{*},
$$

where $\lambda$ is the extrapolation length in $\mathrm{m}$.

On the other hand, we can also formulate the model of motion of $180^{\circ}$ domain wall separating two domains with opposite polarization orientation. Here we can assume that polarization switching is induced by a linearly increasing or trapezoidal electric field. The model needs the initial value of polarization distribution to be specified, for instance, with the use of approximation $P_{0}(x)=P_{0} \tanh (x \backslash \eta)$, where $\eta$ is the parameter related to the thickness of ferroelectric domain boundary.

Notice also that different variations of the fractional time-dependent Landau - Ginzburg equation (the so-called TDLG equation) have been in the focus of several research efforts. Such modification was first proposed by H. Weitzner in [41], and then developed by V.E. Tarasov in [42]. In a study by L. Li and co-authors in [43], a theoretical analysis of the existence and uniqueness of a weak solution to the TDLG equation has been carried out, and the existence of global attractors has been shown. Basically, the detailed study of the Landau - Ginzburg - Devonshire - Khalatnikov equation presents a separate research subproblem and it is beyond the scope of present study.

In general, the fractional cubic-quintic equation (5) can be referred to as semilinear equations of anomalous diffusion, specifically, subdiffusion equations. The construction of computational algorithms for the implementation of the mathematical model, due to their specificity, requires the application of numerical methods.

\section{Numerical techniques}

Current section presents computational aspects of model implementation with a focus on construction of the numerical algorithm based on joint application of a finite-difference method and an iterative procedure to solve a time-fractional semilinear PDE.

In order to derive a computational algorithm, let us reformulate the mathematical problem statement in the following generalized view:

$$
\begin{gathered}
\frac{\partial^{\alpha} u}{\partial t^{\alpha}}=\kappa \frac{\partial^{2} u}{\partial x^{2}}+\varphi(u)+f(x, t), \quad 0<x<L, \quad 0<t \leq t_{o b}, \\
\left.u\right|_{t=0}=u_{0}(x), \quad 0<x<L,
\end{gathered}
$$




$$
-\left.\frac{d u}{d x}\right|_{x=0}+\left.q u\right|_{x=0}-g=0,\left.\quad \frac{d u}{d x}\right|_{x=L}+\left.q u\right|_{x=L}-g=0, \quad 0<t \leq t_{o b},
$$

where $u=u(x, t)$ is unknown function, $\varphi(u)=a u+b u^{3}-c u^{5}$ is semilinear reaction part of the time-fractional PDE, $f(x, t)$ is the source function, $a, b, c, \kappa, q, g$ are positive constants, $\alpha$ is the order of time-fractional derivative.

In present study we consider time-fractional derivative in Caputo sense, namely the left-hand side Caputo's fractional derivative defined as

$$
{ }_{a+}^{C} D_{t}^{\alpha} f(t)=\frac{1}{\Gamma(n-\alpha)} \int_{a}^{t} \frac{f^{(n)}(\xi) d \xi}{(t-\xi)^{\alpha-n+1}},
$$

where $n=[\alpha]+1, \quad \alpha>0, \quad[\alpha]$ is the integer part of $\alpha, 0 \leq\{\alpha\}<1$ is the fractional part of $\alpha$.

In order to derive computational scheme, we apply here the following finitedifference approximation of the Caputo time derivative on the grid $\Omega^{\Delta t}=$ $\left\{t^{j}=j \Delta t, \quad j=\overline{0, M}\right\}:$

$$
{ }^{C} D_{t}^{\alpha} f\left(t^{j}\right)=\frac{1}{\Gamma(2-\alpha) \Delta t^{\alpha}} \sum_{l=0}^{j} \mu_{l} f^{j-l}+O\left(\Delta t^{2}\right),
$$

where the weight functions $\mu_{l}$ are given as follows:

$$
\begin{aligned}
& \mu_{0}=1-\zeta(\alpha-1) \\
& \mu_{1}=2^{1-\alpha}-2+2 \zeta(\alpha-1), \\
& \mu_{2}=3^{1-\alpha}-2 \cdot 2^{1-\alpha}+1-\zeta(\alpha-1), \\
& l=\overline{3, j-1}: \quad \mu_{l}=(l+1)^{1-\alpha}-2 l^{1-\alpha}+(l-1)^{1-\alpha}, \\
& l=j: \quad \mu_{l}=(l-1)^{1-\alpha}-l^{1-\alpha},
\end{aligned}
$$

$\zeta$ is the Riemann zeta function.

The considered approximation has been applied previously to solve the initial-boundary problem for linear time-fractional PDE in [44]. Here we expand this approach to solve the semilinear problem (9)-(11). Let the spacetime grid $\Omega_{\Delta x}^{\Delta t}=\left\{x_{i}=i \Delta x, \quad i=\overline{0, N}, \quad t^{j}=j \Delta t, \quad j=\overline{0, M}\right\}$ covers the computational domain, where $\Delta x$ is the coordinate step and $\Delta t$ is the time step. Based on the introduced approximation of temporal derivative (12) and taking into account the centered finite-difference approximation of the spatial derivative, we arrive at the general approximation for equation (9):

$$
\frac{1}{\Delta t^{\alpha} \Gamma(2-\alpha)} \sum_{l=0}^{j} \mu_{l} u_{i}^{j-l}=\frac{\kappa}{\Delta x^{2}}\left(u_{i+1}^{j}-2 u_{i}^{j}+u_{i-1}^{j}\right)+\varphi_{i}^{j}+f_{i}^{j},
$$

where $i=\overline{1, N-1}, \quad j=\overline{1, M}$. 
Therefore the implicit scheme for the numerical solution of the problem (9)-(11) can be written as:

$$
\begin{gathered}
-\frac{\kappa}{\Delta x^{2}} u_{i-1}^{j}+\left(\frac{\mu_{0}}{\Delta t^{\alpha} \Gamma(2-\alpha)}+\frac{2 \kappa}{\Delta x^{2}}-\left(a+b\left(u_{i}^{j}\right)^{2}-c\left(u_{i}^{j}\right)^{4}\right)\right) u_{i}^{j}-\frac{\kappa}{\Delta x^{2}} u_{i+1}^{j}= \\
=-\frac{1}{\Delta t^{\alpha} \Gamma(2-\alpha)} \sum_{l=1}^{j} \mu_{l} u_{i}^{j-l}+f_{i}^{j}
\end{gathered}
$$

where $i=\overline{1, N-1}, \quad j=\overline{1, M}$.

As far as we solve the cubic-quintic PDE, we obtain a nonlinear system of equations on each time layer. In this case we can combine the finite difference scheme with the iterative procedure. This means that we form a sequence of approximations $u_{i}^{(s)}$ converging to $u_{i}^{j}$ for each time moment $t^{j}, j=\overline{1, M}$, using the formulas: $\left(u_{i}^{(s)}\right)^{3} \approx\left(u_{i}^{(s-1)}\right)^{2} u_{i}^{(s)},\left(u_{i}^{(s)}\right)^{5} \approx\left(u_{i}^{(s-1)}\right)^{4} u_{i}^{(s)}$, where $s=1,2, \ldots$ is the number of iteration. The iterative algorithm at the $j$ time step starts by estimating an initial value of function using the value from the previous time step, that is $u_{i}^{(0)}=u_{i}^{j-1}$. Note that the combination of finite difference method with an iterative scheme allows one to keep the level of accuracy corresponding to the order of approximation of the applied numerical method $[45,46]$.

To approximate the boundary conditions (11) with the second order of accuracy, the asymmetric finite-difference formulas can be used

$$
-\frac{-3 u_{0}^{j}+4 u_{1}^{j}-u_{2}^{j}}{2 \Delta x}+q u_{0}^{j}-g=0, \quad \frac{3 u_{N}^{j}-4 u_{N-1}^{j}+u_{N-2}^{j}}{2 \Delta x}+q u_{N}^{j}-g=0,
$$

where $j=\overline{1, M}$.

The initial condition (10) is also included into the computational scheme. The general system of algebraic equations is solved by Gauss method for each time layer.

Using the approximation, we can write the local truncation error $R_{i}^{j}$ of the difference scheme (13):

$$
\begin{gathered}
R_{i}^{j}=\frac{\Delta t^{-\alpha}}{\Gamma(2-\alpha)} \sum_{l=0}^{j} \mu_{l} u_{i}^{j-l}-\frac{\kappa}{\Delta x^{2}}\left(u_{i+1}^{j}-2 u_{i}^{j}+u_{i-1}^{j}\right)-\varphi_{i}^{j}-f_{i}^{j}= \\
=\frac{\Delta t^{-\alpha}}{\Gamma(2-\alpha)} \sum_{l=0}^{j} \mu_{l} u_{i}^{j-l}-{ }^{C} D_{t}^{\alpha} u\left(x_{i}, t^{j}\right)-\kappa\left(\frac{u_{i+1}^{j}-2 u_{i}^{j}+u_{i-1}^{j}}{\Delta x^{2}}-\frac{\partial^{2} u\left(x_{i}, t^{j}\right)}{\partial x^{2}}\right)= \\
=O\left(\Delta t^{2}\right)+\kappa O\left(\Delta x^{2}\right)=O\left(\Delta t^{2}+\Delta x^{2}\right) .
\end{gathered}
$$

Presently, researchers are paying more and more attention to finding a solution to the fractional Landau - Ginzburg equation using numerical methods. In particular, using grid methods, weighted residuals, finite elements, etc. Accordingly, the stability and convergence of the obtained schemes are investigated elsewhere [47-49]. Analysis of a numerical solution based on the implicit 
scheme (12) for linear fractional subdiffusion equation has been shown in [44]. Basically, the computational scheme constructed for numerical solution of the semilinear fractional PDE requires an additional theoretical analysis.

It should be pointed that our approach involves modeling of a complex dynamics of physical system based on numerical solution of the time-fractional PDE representing a modification of the thermodynamic model, whereas the studies $[28,24,50,51]$ have proposed the fractional model of ferroelectric hysteresis based on the numerical approximation of fractional derivatives.

\section{Computer simulation results and discussion}

In this section, we apply the constructed numerical scheme for the computer simulation of polarization switching in ferroelectric thin films with the first order phase transition.

\subsection{Computational experiments setup}

The derived numerical algorithms were implemented using Matlab programming platform. The application program interface is intended for modeling of the space-time distribution of polarization and polarization-electric field dependence during the process of ferroelectric switching. To perform simulations, we need to initialize characteristics of the material (thermodynamic parameters, the thickness of the ferroelectric sample), the observation time, and the applied field. The number of nodes of the space-time grid covering computational domain are also need to be specified.

The most studied case of polarization switching is $180^{\circ}$ polarization inversion, in which an external field is applied along the spontaneous polarization axis and its orientation changes directly by $180^{\circ}$. For example, $180^{\circ}$ polarization switching is observed for almost all uniaxial ferroelectric crystals, such as triglycine sulfate $\left(\mathrm{NH}_{2} \mathrm{CH}_{2} \mathrm{COOH}\right)_{3} \mathrm{H}_{2} \mathrm{SO}_{4}$, potassium dihydrogen phosphate $\mathrm{KH}_{2} \mathrm{PO}_{4}$, lithium niobate $\mathrm{LiNbO}_{3}$, etc. Polarization switching process can be realized by means of two relevant mechanisms, namely nucleation and growth of $180^{\circ}$ domains with spontaneous polarization parallel to applied field as well as lateral expansion of oppositely orientated domains.

Although the generalized Landau - Khalatnikov model (5) - (8) is most suitable for uniaxial crystals, it can also be used to describe polarization switching in multiaxial crystals. In particular, barium titanate $\left(\mathrm{BaTiO}_{3}\right)$ has both $90^{\circ}$ and $180^{\circ}$ oriented types of domain structures in the tetragonal phase at room temperature. Using an appropriate external field applied along the $c$-oriented domains of $\mathrm{BaTiO}_{3}, 180^{\circ}$ polarization switching can be observed $[29,52,53]$.

In the current numerical experiments, we consider switching of ferroelectrics under the periodic electric field (2). The simplified scheme of domain structure and $180^{\circ}$ polarization switching in $c$-domains of $\mathrm{BaTiO}_{3}$ is illustrated in 


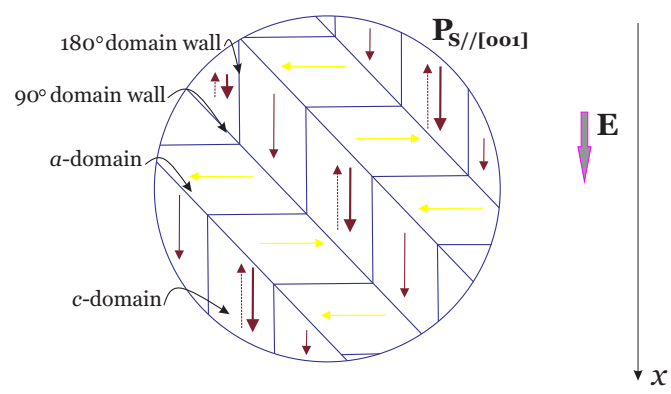

Fig. 1 The sketch of ferroelectric domain structure and $180^{\circ}$ polarization switching in c-domains of $\mathrm{BaTiO}_{3}$.

Table 1 The temperature dependent coefficients of the free energy for barium titanate

\begin{tabular}{lll}
\hline Name & Numerical value & Unit \\
\hline$A / 2$ & $-3.34(T-381) \cdot 10^{5}$ & $\mathrm{~m} / \mathrm{F}$ \\
$B / 4$ & $-(3.6(T-448)-202) \cdot 10^{6}$ & $\mathrm{~m}^{5} /\left(\mathrm{C}^{2} \cdot \mathrm{F}\right)$ \\
$C / 6$ & $(5.52(T-120)+276) \cdot 10^{7}$ & $\mathrm{~m}^{9} /\left(\mathrm{C}^{4} \cdot \mathrm{F}\right)$ \\
\hline
\end{tabular}

Figure 1. The initial polarization is associated with the original equilibrium state of ferroelectric, that equals zero at the start time moment $t=0$. Here we assume that the complete $180^{\circ}$ switching event is induced by an applied field. The intermediate polarization states during the switching process match to range of field variation $\left(-E_{0}, E_{0}\right)$.

Table 1 lists the values of thermodynamic parameters for barium titanate at temperature $T$ (in K) $[29,54]$ used for computer simulations. The parameters of the computational experiments correspond to the experimental data described in [55]. In the tests, the parameters are set to be: the amplitude of the field intensity is $E_{0}=2.5 \cdot 10^{5} \mathrm{~V} / \mathrm{m}$, the field frequency is $f=50 \mathrm{~Hz}(\omega=2 \pi \cdot f)$, the gradient coefficient is $\psi=5 \cdot 10^{-8} \mathrm{~m}^{3} / \mathrm{F}$, the kinetic coefficient (which determines the velocity of movement of domain walls) is $\delta=2 \cdot 10^{5} \mathrm{~m} \cdot \mathrm{s} / \mathrm{F}[56]$. The observation time is $\theta=0.03 \mathrm{~s}$, the characteristic time of the process is $t^{*}=1 \mathrm{~s}$, and the sample thickness is varied.

By the above, to visualize the polarization-electric field dependence for each time moment, we calculate the averaged value of the polarization over the crystal thickness:

$$
P(t)=\frac{1}{L} \int_{0}^{L} P(x, t) d x
$$

\subsection{Numerical convergence study}

In order to verify the obtained simulation results, we applied several approaches. First we performed the comparison of numerical solutions with exact 


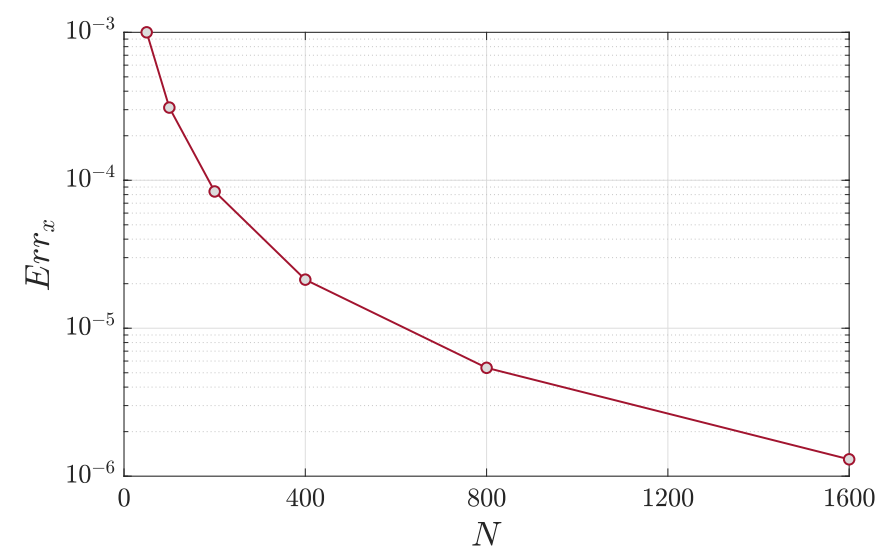

Fig. 2 The relative error (in a logarithmic scale) vs. the number of spatial partitions.

solutions for test linear fractional problems. Further, when solving nonlinear fractional problems, the verification of the simulation results was realized by means of comparison of the simulation result with the data of an integer analogue of the model in the limiting modes (e.g. at $\alpha=0.999$ ). Finally, as we will show further on, the adequacy of the results are established by comparing of simulation data with experimental observations of polarization hysteresis in ferroelectrics.

In addition, we conducted a study of the practical convergence of the scheme for numerical solution of the fractional Landau - Ginzburg - Devonshire - Khalatnikov equation. For this purpose, we applied the testing scheme by the double-counting procedure in two versions. The numerical convergence study is based on refining the spatial and temporal steps of the grid covering solution domain separately. This approach is widely used for numerical testing schemes in terms of the study of their convergence rate $[57,58]$.

In the first case, we estimate the spatial relative error using $l_{2}$-norm as follows:

$$
\operatorname{Err}_{x}=\frac{\|\hat{P}-\tilde{P}\|_{2}}{\|\hat{P}\|_{2}},
$$

where $\hat{P}(x, t)$ is the polarization calculated at the spatial partitions number $N$ and the polarization $\tilde{P}(x, t)$ corresponds to the doubled number of spatial partitions $2 N$ with the number of time partitions $M=500$.

The test results are shown in Figure 2. The computations were performed for the set of parameters mentioned above, the simple thickness is defined to be $1 \mu \mathrm{m}$, and the order of the fractional derivative equals 0.9 . The observation period is set to be $\theta=0.03 \mathrm{~s}$. Hence, we can conclude that the error decreases rapidly when the spatial grid is refined. A sufficiently acceptable accuracy (of the order of $0.01 \%$ ) can be obtained using a grid with the number of spatial partitions $N=100-200$. 


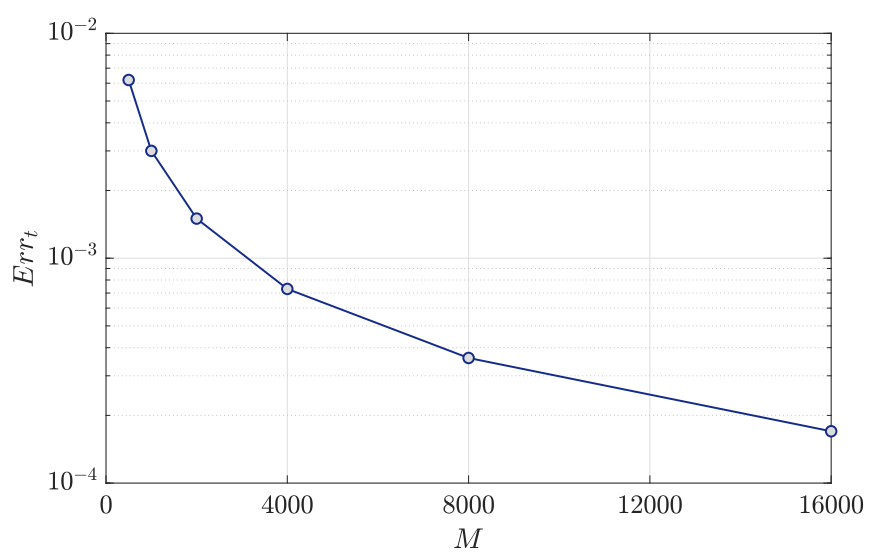

Fig. 3 The relative error (in a logarithmic scale) vs. the number of time partitions.

Further, the time relative error is calculated by the formula:

$$
\operatorname{Err}_{t}=\frac{\|\bar{P}-\check{P}\|_{2}}{\|\bar{P}\|_{2}}
$$

where $\bar{P}(x, t)$ is the solution obtained with the number of time partitions $M$ and $\check{P}(x, t)$ is associated with the doubled number of time partitions $2 M$.

Computations were performed at $N=200$, while the observation period is also specified as $\theta=0.03 \mathrm{~s}$. The dependence of relative error on the number of time partitions is illustrated in Figure 3. We stress that a decrease in the time step also leads to a decreasing the error, but in terms of accuracy the numerical solution turns out to be more sensitive to a change in the time step compared to the spatial step. So, with the number of partitions $M=4000$, the relative error is estimated to be $0.05 \%$.

As a result, although the use of a refined grid (for example, with the partitions number $N=1600$ ) corresponds to an error of the order of $10^{-6} \%$, does not provide benefit in terms of accuracy, because the total error will be limited by the error caused by the time sampling. The mesh refinement is always associated with an increase in the computational time, for an already resourceintensive fractional-differential problem. In computational experiments when controlling the prior accuracy (for example, $0.01 \%$ ), it makes sense to take the optimal ratio of steps in space and time as $M=2000, N=200$.

The iterative procedure included into general algorithm was also examined to verify the numerical convergence. In practice, three or four iterations on each time layer were enough to get accuracy sufficient compared to the total accuracy of the numerical solution (in this case ratio error is approximately equal to $0.001 \%$ ). 


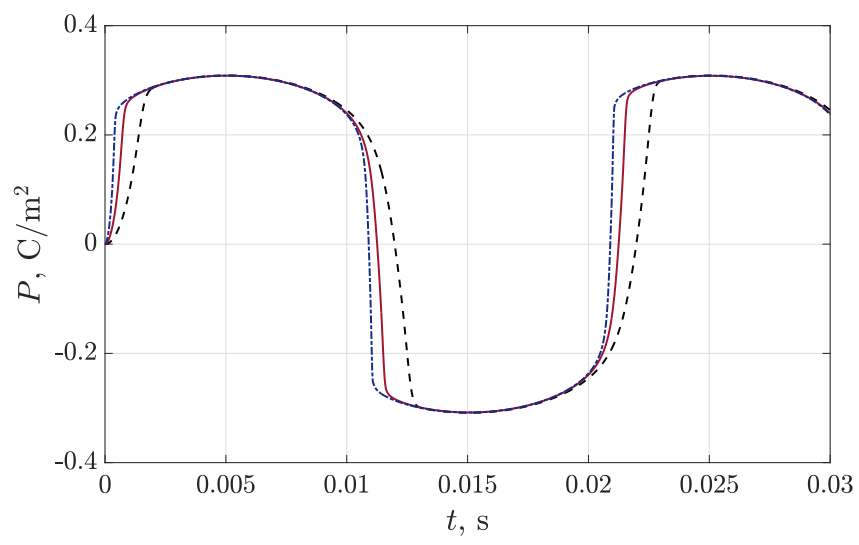

Fig. 4 The time dependence of the averaged polarization during the polarization switching in $\mathrm{BaTiO}_{3}$ thin film for the integer analogue of the model (dashed line) and under variation of the fractional derivative order: $\alpha=0.85$ (solid line), $\alpha=0.75$ (dash-dotted line).

\subsection{Simulation of ferroelectric polarization switching in $\mathrm{BaTiO}_{3}$ thin films}

Let us consider the results of computational experiments on the example of modeling of the characteristics of the polarization switching in $c$-domains of barium titanate thin film, that is induced by sinusoidal electric field $E(t)$. Figure 4 shows the simulation results of the dynamics of the averaged polarization over the crystal thickness $P(t)$ during the polarization switching. The ferroelectric hysteresis loops as polarization - electric field functional dependencies $P(E)$ are demonstrated in Figure 5. The simulation results are visualized for different values of order of fractional derivative $\alpha$ compared to the simulation result obtained by integer Landau - Khalatnikov model. The experimental data reported in [55] is also plotted in Figure 5.

In a series of computational experiments, the value of the fractional derivative order $\alpha$ was varied from 0.75 to 1 with a step of 0.05 .

From the results obtained, it can be concluded that a decrease in the order of the time fractional derivative corresponds to a narrowing of the dielectric hysteresis loop while maintaining its shape. The best agreement with the experimental data corresponds to the order of fractional derivative $\alpha=0.85$ (with a standard deviation of 0.027 ).

In this instance, the observations indicate that the main characteristics of the hysteresis loop, such as coercive field $E_{c}=0.95 \cdot 10^{5} \mathrm{~V} / \mathrm{m}$ and residual polarization $P_{r}=0.24 \mathrm{C} / \mathrm{m}^{2}$ coincide with the data described in the literature (e.g., $[29,54,56,59,60])$.

It can be noted that one of the important Landau model parameter is the scaling factor for the field $\nu$, which has to be adapted for simulation of polarization hysteresis ferroelectrics in high frequency fields. An increase in this coefficient results in a narrowing of the hysteresis loop and its extension along the ordinate axis. Thus, the order of fractional differentiation $\alpha$ as a numeri- 


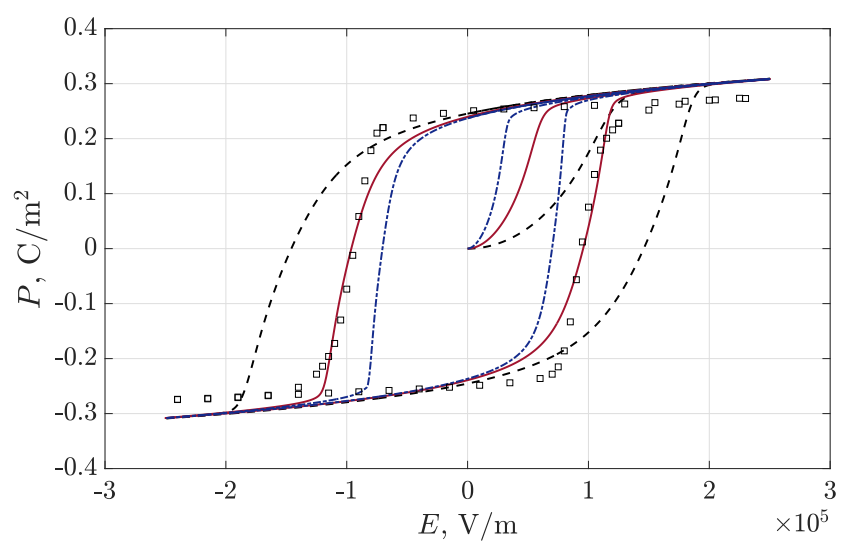

Fig. 5 The polarization - electric field hysteresis dependencies for $\mathrm{BaTiO}_{3}$ thin film, calculated for the integer analogue of the model (dashed line) and under variation of the fractional derivative order: $\alpha=0.85$ (solid line), $\alpha=0.75$ (dash-dotted line), compared with experimental data [55].

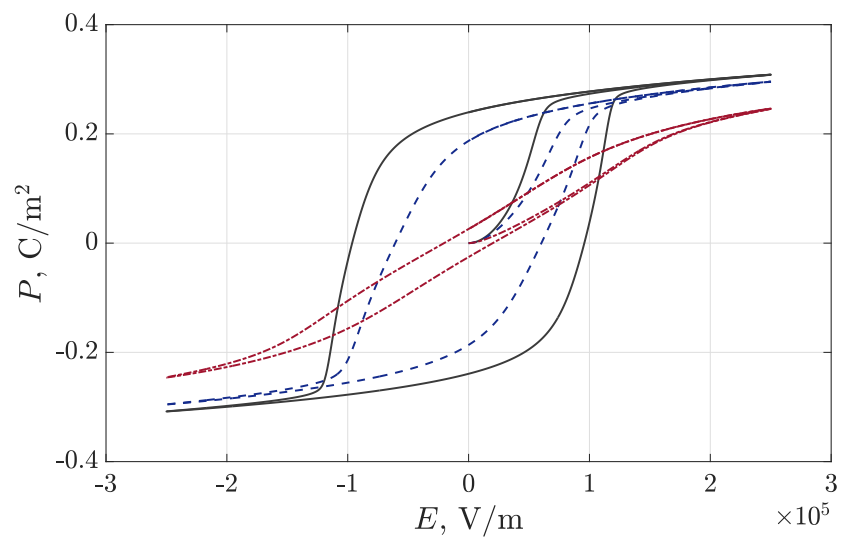

Fig. 6 Polarization - electric field hysteresis dependencies for $\mathrm{BaTiO}_{3}$ with the different values of sample thickness: $L=1 \mu \mathrm{m}$ (solid curve), $L=10 \mathrm{~nm}$ (dashed line), $L=5 \mathrm{~nm}$ (dash-dotted line).

cal characteristic of the memory effect, is an additional control parameter of the fractional model of polarization switching, which can be tuned to provide better agreement with the experimental data.

In the aspect of studying the influence of the values of control parameters on the behavior of the simulated characteristics, further we consider the so-called "size effect", which is presented here by the dependence of the characteristics of ferroelectric hysteresis on the sample thickness. Figure 6 shows the hysteresis loops calculated by means of the generalized Landau - Khalatnikov model with varying the thickness of $\mathrm{BaTiO}_{3}$. 
Numerical experiments indicate that the shape of the hysteresis loop does not depend on the crystal thickness $L$ for bulk materials. As known, in this case the generalized Landau - Khalatnikov model can be reduced to the case of the classical model described by the Cauchy problem for an ordinary differential equation (the gradient term can be neglected for bulk objects). At the same time, for low-dimensional structures (nanowires, thin films, island coatings, etc.), taking into account the Ginzburg term and introducing Robin boundary conditions are relevant for simulations.

We can observe a significant distortion of the hysteresis loop with a decrease in the film thickness, such as deformation, narrowing of the loop, formation of the double loop. In our computational experiments, the film thickness of $3 \mathrm{~nm}$ is estimated to be critical, since the hysterisis loop disappears at this thickness. These results agree with the data obtained for nanowires and thin films of barium titanate by independent authors in $[61,62]$.

\section{Conclusion}

In this paper we performed the detailed numerical study of the fractional modification of Landau - Ginzburg - Devonshire - Khalatnikov model proposed to describe the polarization switching in ferroelectrics. To solve time fractional cubic-quintic PDE, we constructed the computational scheme based on the combination of a finite difference approximation of Caputo derivative and an iterative procedure. The computational algorithm was implemented in Matlab to perform numerical simulations of ferroelectric hysteresis dependencies of polarization on applied field. In order to verify simulation results, the numerical convergence study was conducted. As a result, this allowed us to estimate the optimal values of computational parameters for computational experiments.

We demonstrated simulation results on the example of barium titanate thin film. The polarization - electric field dependencies for $\mathrm{BaTiO}_{3}$ thin films were calculated by means of fractional Landau - Ginzburg - Devonshire Khalatnikov model. The results of computational experiments were demonstrated at varying the order of fractional derivative. The observations suggest that the possibility of varying the order of fractional differentiation provides the results of the implementation of the differential model, which better reproduce the experimentally observed regularities in comparison with the integer analogues. As a numerical characteristic of the memory effect, the order of fractional derivative can be used as an additional control parameter of the dynamic model that can be tuned to ensure optimal agreement with the experimental data.

Thus, we can conclude that the fractional generalization of the Landau Khalatnikov model formalized the dynamic responses of ferroelectrics under external exposure significantly expands the range of functional capabilities of the numerical modeling methodology as applied to the study of this class of fractal physical systems with memory. 
Acknowledgements This work was supported by the Russian Foundation for Basic Research (project no. 20-31-90075).

\section{Conflict of interest}

The authors declare that they have no conflict of interest.

\section{References}

1. Kilbas, A.A., Srivastava, H.M., Trujillo J.J.: Theory and applications of fractional differential equations. North Holland Mathematics Studies, Elsevier Science, Publishers BV, Amsterdam (2006)

2. Bagley, R.L., Peter J.T.: On the fractional calculus model of viscoelastic behavior. Journal of Rheology 30, 133-135 (1986)

3. Hilfer, R.: Applications of fractional calculus in physics. World Scientific Publishing Company, Singapore (2000)

4. Carpinteri A., Mainardi F.: Fractals and fractional calculus in continuum mechanics. Springer, Vienna (1997)

5. Uchaikin, V.V.: Fractional derivatives for physicists and engineers: background and theory. Higher Education Press, Beijing (2013)

6. Barnsley, M., Demko, S.: Chaotic dynamics and fractals. Academic Press, New York (1986)

7. Takayasu, H.: Fractals in the physical sciences. Manchester University Press, Manchester (1990)

8. Uchino, K.: Fractal phenomena in ferroelectrics. J. Nanotech. Mater. Sci. 1, 12-26 (2014)

9. Ozaki, T., Fujii, K., Ohgami J.: Fractal aspects of lamellar ferroelectric domain structures formed under the influence of depolarization fields in $\mathrm{CsH}_{2} \mathrm{PO}_{4}$ and $\left(\mathrm{NH}_{2} \mathrm{CH}_{2} \mathrm{COOH}\right)_{3} \mathrm{H}_{2} \mathrm{SO}_{4}$. JPSJ 64, 2282-2285 (1995)

10. Shur,V.Ya., Kuznetsov, D.K., Lobov, A.I., Nikolaeva, E.V., Dolbilov, M.A., Orlov, A.N., Osipov, V.V.: Formation of self-similar surface nano-domain structures in lithium niobate under highly nonequilibrium conditions. Ferroelectrics 341(1), 85-93 (2006)

11. Jeng, Y.-R., Tsai,P.-C., Fang T.-H.: Nanomeasurement and fractal analysis of PZT ferroelectric thin films by atomic force microscopy. Microelectron. Eng. 65, 406-415 (2003)

12. Tadic, B.: Switching current noise and relaxation of ferroelectric domains. EPJ B 28, 81-89 (2002)

13. Roy, M.K., Sarkar, S, Dattagupta, S.: Evolution of $180^{c} \mathrm{irc}, 90^{c} \mathrm{irc}$, and vortex domains in ferroelectric films. Applied Physics Letters 95, 192905 (2009)

14. Galiyarova, N.M., Bey, A.B., Kuznetzov, E.A., Korchmariyuk, Y.I.: Fractal dimensionalities and microstructural parameters of piezoceramics PZTNB-1. Ferroelectrics 307, 205-211 (2004)

15. Wu, Z., Duan, W., Wu, J., Gu, B-L.: Self-organization nanodomain structure in ferroelectric ultrathin films. Nanotechnology, 18, 325703-325707 (2007)

16. Kim, S., Park, K-W., Woo, H., Hong, J.: Time-resolved fractal dimension analysis in ferroelectric copolymer thin films using R-based image processing. Material Letters 230, 195-198 (2018)

17. Mitic, V.V., Lazovic, G., Lu, C-A., Paunovic, V., Radovic, I., Stajcic, A., Vlahovic, B.: The nano-scale modified $\mathrm{BaTiO}_{3}$ morphology influence on electronic properties and ceramics fractal nature frontiers. Appl. Sci. 10, 3485(14) (2020)

18. Casals, B., Nataf, G.F., Salje, E.K.: Avalanche criticality during ferroelectric ferroelastic switching. Nat. Commun. 12, 345 (2021)

19. Maslovskaya, A.G., Barabash, T.K.: Fractal model of polarization switching kinetics in ferroelectrics under nonequilibrium conditions of electron irradiation. J. Phys.: Conf. Ser. 973, 012038(11) (2018)

20. Meilanov, R.P., Sadykov, S.A.: Fractal model for polarization switching kinetics in ferroelectric crystals. Technical Physics 44(5), 595-597 (1999) 
21. Galiyarova, N.: Fractal dielectric response of multidomain ferroelectrics from the irreversible thermodynamics standpoint. Ferroelectrics 222(1), 381-387 (1999)

22. Galiyarova, N., Korchmaryuk, Ya.: Response of some fractal nonlinear systems, Ferroelectrics, 222(1), 389-395 (1999)

23. Weron, K., Klauzer, A.: Probabilistic basis for the Cole-Cole relaxation law. Ferroelectrics 236(1), 59-69 (2000)

24. Ducharne, B., Sebald, G., Guyomar, D.: Time fractional derivative for frequency effect in ferroelectrics. Proc. 18th IEEE International Symposium on the Applications of Ferroelectrics, Xian 1-4 (2009)

25. Meng, R.: Application of fractional calculus to modelling the non-linear behaviors of ferroelectric-polymer composites: Viscoelasticity and Dielectricity. Membranes 11(6) 409 (2021)

26. Maslovskaya, A., Barabash, T.: Fractal parameterization analysis of ferroelectric domain structure evolution induced by electron beam irradiation. Proc. IOP Conf. Series: Materials Science and Engineering 168, 012028(6) (2017)

27. Moroz, L.I., Maslovskaya, A.G.: Hybrid stochastic fractal-based approach to modeling the switching kinetics of ferroelectrics in the injection mode. Math. Models. Comput. Simul. 12, 348-356 (2020)

28. Blinc, R., Zeks, B.: Soft modes in ferroelectrics and antiferroeletrics. North-Holland Publ.Co., Amsterdam (1974)

29. Rabe, K.M., Ahn, C., Triscone, J.: Physics of ferroelectrics : a modern perspective. Springer, Berlin (2007)

30. Omura, M., Adachi, H., Ishibashi, Y.: Simulations of ferroelectric characteristics using a onedimensional lattice model. Jpn. J. Appl. Phys. 30, 2384-2387 (1991)

31. Wang, C.L., Zhang, L., Zhong, W.L., Zhang P.L.: Switching characters of asymmetric ferroelectric films. Phys. Lett. A 254, 297-300 (1999)

32. Srinoi, S. , Laosiritaworn, Y.: Investigation of temperature-driven ferroelectric phasetransition via modified Heisenberg model: the Monte-Carlo simulation. Adv. Mater. Res. 813, 315--318 (2013)

33. Ouyang, K., Ren T.-L., Liu, L.-T., Wei D.: Simulation on the hysteresis of ferroelectric thin films. Integrated Ferroelectrics 64(1), 69-75 (2004)

34. Song, T.K., Kim, J.S., Kim, M.H., Lim, W., Kim, Y.S., Lee,J.C.: Landau - Khalatnikov simulations for the effects of external stress on the ferroelectric properties of $\mathrm{Pb}(\mathrm{Zr}, \mathrm{Ti}) \mathrm{O}_{3}$ thin films. Thin Solid Films 424, 84-87 (2003)

35. Song, T.K.: Landau -- Khalatnikov simulations for ferroelectric switching in ferroelectric random access memory application. J. Korean Phys. Soc. 46(1), 5-9 (2005)

36. Starkov, A.S., Pakhomov, O.V., Starkov, I.A.: Effect of thermal phenomena on a secondorder phase transition in the Landau -- Ginzburg model. J. Exper. Theor. Phys. Lett. 91(10), 507-511 (2010)

37. Maslovskaya, A.G., Moroz, L.I., Chebotarev, A.Yu., Kovtanyuk, A.E.: Theoretical and numerical analysis of the Landau - Khalatnikov model of ferroelectric hysteresis. Commun. Nonlinear SCI 93, 105524(13) (2021)

38. Petráš, I.: Fractional-order nonlinear systems: modeling, analysis and simulation. Springer, Dordrecht (2010)

39. Podlubny, I.: Fractional differential equations. Academic press, San Diego (1999)

40. Moroz, L., Maslovskaya, A.: Computational techniques for modeling time-fractional dynamics of polarization switching in ferroelectrics. Proc. of the CEUR "Workshop Proceedings", 2783, 180-191 (2020)

41. Weitzner, H., Zaslavsky, G.M.: Some applications of fractional derivatives. Commun. Nonlinear. Sci. Numer. Simul. 8, 273-281 (2003)

42. Tarasov, V.E., Zaslavsky, G.M.: Fractional Ginzburg — Landau equation for fractal media. Physica A: Statistical Mechanics and its Applications 354, 249-261 (2005)

43. Li, L., Jin, L., Fang S.: Large time behavior for the fractional Ginzburg — Landau equations near the BCS-BEC crossover regime of Fermi gases. Bound. Value Probl. 8(16), $1-16$ (2017)

44. Dimitrov, Y.: A second order approximation for the Caputo fractionak derivative. J. Fractional Calc. Appl.7, 175-195 (2016) 
45. Chebotarev, A.Yu., Kovtanyuk, A.E., Grenkin, G.V., Botkin, N.D., Hoffmann, K.-H.: Nondegeneracy of optimality conditions in control problems for a radiative-conductive heat transfer model. Appl. Math. Comput. 289, 371-380 (2016)

46. Chebotarev, A.Yu., Grenkin, G.V., Kovtanyuk, A.E., Botkin, N.D., Hoffmann K.-H.: Diffusion approximation of the radiative-conductive heat transfer model with Fresnel matching conditions, Comm. Nonlinear Sci. Num. Simulat. 57, 290-298 (2018)

47. Li, M., Huang, C., Wang,N.: Galerkin finite element method for the nonlinear fractional Ginzburg-Landau equation. Appl. Num. Math. 118, 131-149 (2017)

48. Yang, Y., Tao, J., Zhang, S., Sivtsev, P.: A Jacobi collocation method for the fractional Ginzburg-Landau differential equation. Adv. Appl. Math. Mech. 12, 57-86 (2019)

49. Zaky, M.A., Hendy, A.S., Staelen, R.H.: Alikhanov Legendre-Galerkin spectral method for the coupled nonlinear time-space fractional Ginzburg-Landau complex system. Mathematics 9, 183 (2021)

50. Guyomar, D., Ducharne,B., Sébald G.: Time fractional derivatives for voltage creep in ferroelectric materials: theory and experiment. J. Phys. D: Appl. Phys. 41, 125410 (2008)

51. Ducharne, B., Newell, B., Sebald, G.: Unique fractional derivative operator to simulate all dynamic piezoceramic dielectric manifestations: from aging to frequency-dependent hysteresis. IEEE Trans. Ultrason. Ferroelectr. Freq. Control 67(1) 197-206 (2020)

52. Lines, M.E., Glass, A.M.: Principles and applications of ferroelectrics and related materials. Clarendon Press, Oxford (2001)

53. Smolensky, G.A., Bokov, V.A., Yusupov, V.A., Krainik, N.N., Pasynkov, R.E., Sokolov, A.I.: Ferroelectrics and related materials. Gordon and Breach, New York (1984)

54. Hlinka, J., Marton, P.: Phenomenological model of $90^{\circ}$ domain wall in $\mathrm{BaTiO}_{3}$-type ferroelectrics. Phys. Rev. B 74, 104104 (2006)

55. Moulson, A., Herbert, J.M.: Electroceramics. Materials, properties, applications. Chapman and Hall, London (1990)

56. Narita, F., Kobayashi, T., Shindo, Ya.: Evaluation of dielectric and piezoelectric behavior of unpoled and poled barium titanate polycrystals with oxygen vacancies using phase field method. Int. J. Smart. Nano. Mater. 7(4), 265-275 (2016)

57. Jeong, D., Li, Y., Lee, C., Yang, J., Choi, Y., Kim, J.: Verification of convergence rates of numerical solutions for parabolic equations. Mathematical Problems in Engineering 2019, $8152136(2019)$

58. Pinto, L., Sousa, E.: Numerical solution of a time-space fractional Fokker Planck equation with variable force field and diffusion. Communications in Nonlinear Science and Numerical Simulation 50 211--228 (2017)

59. Nakamura, K.: Ultrasonic transducers: Materials and design for sensors, actuators and medical applications. Woodhead Publ. Ltd. (2012)

60. Glazkova, E., McCash, K.,Chang, C.-M., Mani, B., Ponomareva, I.: Tailoring properties of ferroelectric ultrathin films by partial charge compensation. Appl. Phys. Lett. 104, 012909 (2014)

61. Hong, J., Fanga, D.: Size-dependent ferroelectric behaviors of $\mathrm{BaTiO}_{3}$ nanowires. Appl. Phys. Lett. 92, 012906 (2008)

62. Su, Y., Chen,H., Li, J., Soh, A.K., Weng, G.J.: Effects of surface tension on the sizedependent ferroelectric characteristics of free-standing $\mathrm{BaTiO}_{3}$ nano-thin films. J. Appl. Phys. 110, 084108 (2011) 